PROCEEDINGS OF THE

AMERICAN MATHEMATICAL SOCIETY

Volume 139, Number 5, May 2011, Pages 1625-1635

S 0002-9939(2010)10778-5

Article electronically published on November 4, 2010

\title{
MAXIMAL UNIVALENT DISKS OF REAL RATIONAL FUNCTIONS AND HERMITE-BIEHLER POLYNOMIALS
}

\author{
VLADIMIR P. KOSTOV, BORIS SHAPIRO, AND MIKHAIL TYAGLOV \\ (Communicated by Ken Ono)
}

\begin{abstract}
The well-known Hermite-Biehler theorem claims that a univariate monic polynomial $s$ of degree $k$ has all roots in the open upper half-plane if and only if $s=p+i q$, where $p$ and $q$ are real polynomials of degree $k$ and $k-1$ respectively with all real, simple and interlacing roots, and $q$ has a negative leading coefficient. Considering roots of $p$ as cyclically ordered on $\mathbb{R} P^{1}$ we show that the open disk in $\mathbb{C} P^{1}$ having a pair of consecutive roots of $p$ as its diameter is the maximal univalent disk for the function $R=\frac{q}{p}$. This solves a special case of the so-called Hermite-Biehler problem.
\end{abstract}

\section{INTRODUCTION}

Rational functions of the form

$$
R=\frac{q}{p}
$$

where $p$ and $q$ have all real, simple and interlacing roots, appear often in different areas of mathematics and enjoy a number of nice properties. Below we discuss a new property of such $R$ related to the classical Hermite-Biehler theorem. Namely, we start with the following question.

Problem 1. For a given real rational function $F$, describe real (i.e. invariant under complex conjugation) disks in $\mathbb{C} P^{1}$ in which $F$ is univalent.

An obvious necessary condition for a real disk $D \subset \mathbb{C} P^{1}$ to be a univalent domain of $F$ is that $F$ restricted to the real interval $D \cap \mathbb{R} P^{1}$ is univalent. The main result of this paper is that for rational functions of the form (1.1) this simple necessary condition is, in fact, sufficient; see Theorem 1.3. It has an immediate implication for the location of roots of Hermite-Biehler polynomials, i.e. polynomials whose roots belong to the open upper half-plane. Denoting the monic polynomial proportional to the latter one by $s$ we get by the Hermite-Biehler theorem that $s=p+i q$, where $p$ and $q$ have all real, simple and interlacing roots and different signs of their leading coefficients. Since the roots of $s$ solve the equation $p+i q=0$ or, equivalently, $\frac{q}{p}=i$,

Received by the editors May 4, 2010.

2010 Mathematics Subject Classification. Primary 26C05; Secondary 30C15.

Key words and phrases. Hermite-Biehler theorem, root localization.

The third author was supported by the Sofja Kovalevskaja Research Prize of the Alexander von Humboldt Foundation.

(C)2010 American Mathematical Society 
we can apply Theorem 1.3 to restrict the location of roots of $s$. Additionally, our result gives a partial answer to the following general Hermite-Biehler problem as formulated in [5], p. $575[1]$

Question. Given a pair of real polynomials $(p, q)$, give restrictions on the location of the roots of $p+i q$ in terms of the location of the roots of $p$ and $q$.

In what follows we will assume for simplicity that $p$ and $q$ are monic polynomials, which does not affect any of the results below. We start with a certain localization result for the critical points of $R$ given by (1.1). For a given pair $(p, q)$ of polynomials, define its Wronskian as

$$
W(p, q)=p^{\prime} q-q^{\prime} p .
$$

Properties of the Wronski map were studied in detail in e.g. [3] and [4. Notice that if $p$ and $q$ are coprime, then the roots of $W(p, q)$ are exactly the critical points of $R=\frac{q}{p}$. (Here $R$ is considered as a map of $\mathbb{C} P^{1}$ to itself.) In the case when $p$ and $q$ have all real, simple and interlacing roots, one can easily see that all roots of $W(p, q)$ are non-real. (The latter fact was already known to C. F. Gauß.)

Our first result concerns the location of these roots. Assume that $p$ and $q$ are real polynomials of degrees $k$ and $k-1$ respectively with all real, simple and interlacing roots and let $p_{1}<p_{2}<\ldots<p_{k}$ be the roots of $p$. Denote by $D_{j}, j=1, \ldots, k-1$ the open disk bounded by the circle $C_{j} \subset \mathbb{C}$ having the pair $\left(p_{j}, p_{j+1}\right)$ as its diameter and denote by $D_{0}$ the open disk bounded by the circle $C_{0} \subset \mathbb{C}$ having the pair $\left(p_{1}, p_{k}\right)$ as its diameter. Set $\Omega_{p}=\bar{D}_{0} \backslash \bigcup_{j=1}^{k-1} D_{j}$, where $\bar{D}_{0}$ is the closure of $D_{0}$; see Figure 1. Our first result is as follows.

Theorem 1.1. Under the above assumptions, all roots of $W(p, q)$ lie in $\Omega_{p}$.

A similar statement for the case $q=p^{\prime}$ can be found in 2. Further, note that for any real $\alpha$, the roots of the polynomial $p+\alpha q$ are real and interlacing the roots of the polynomial $q$ (as well as the roots of the polynomial $p$ whenever $\alpha \neq 0$ ). Denoting by $p_{j}(\alpha)$ the roots of the polynomial $p+\alpha q$ consider the open disks $D_{j}(\alpha), j=1, \ldots, k-1$ having the intervals $\left(p_{j}(\alpha), p_{j+1}(\alpha)\right)$ as their diameters and denote by $D_{0}(\alpha)$ the open disk having $\left(p_{1}(\alpha), p_{k}(\alpha)\right)$ as its diameter. Finally, let $\Omega_{p}(\alpha)=\bar{D}_{0}(\alpha) \backslash \bigcup_{j=1}^{k-1} D_{j}(\alpha)$. Since for any real $\alpha$ one has $W(p+\alpha q, q)=W(p, q)$, Theorem 1.1 implies the following.

Corollary 1.2. All roots of $W(p, q)$ lie in $\bigcap_{\alpha \in \mathbb{R}} \Omega_{p}(\alpha)$.

Since in our case all roots of $W(p, q)$ are non-real, it is natural to ask if every real polynomial of even degree with all non-real roots can be represented as the Wronskian of a suitable pair $(p, q)$ as above. Consider the space $\operatorname{Int}_{k}$ of all pairs $(p, q)$ where $p=z^{k}+a_{1} z^{k-2}+\ldots+a_{k-1}, q=z^{k-1}+b_{1} z^{k-2}+\ldots+b_{k-1}$ with all real, simple and interlacing roots. The next statement follows from the main result of [10, which was conjectured by J. Milnor.

Proposition 1. The Wronski map $W$ defines a diffeomorphism of the space Int $_{k-1}$ and the space Pol Pon-2 $_{2 k}$ consisting of polynomials $u(z)=z^{2 k-2}+c_{1} z^{2 k-3}+\ldots+c_{2 k-2}$ which are strictly positive on the whole real axis $\mathbb{R}$; i.e., it is a bijective map between Int $_{k-1}$ and Pol $_{2 k-2}$ (with everywhere non-vanishing Jacobian).

Finally, the main result of this paper is as follows. Take $R=\frac{q}{p}$ as in (1.1).

\footnotetext{
${ }^{1}$ When preparing this paper we received the sad news that Professor S. Fisk passed away after a long period of illness.
} 

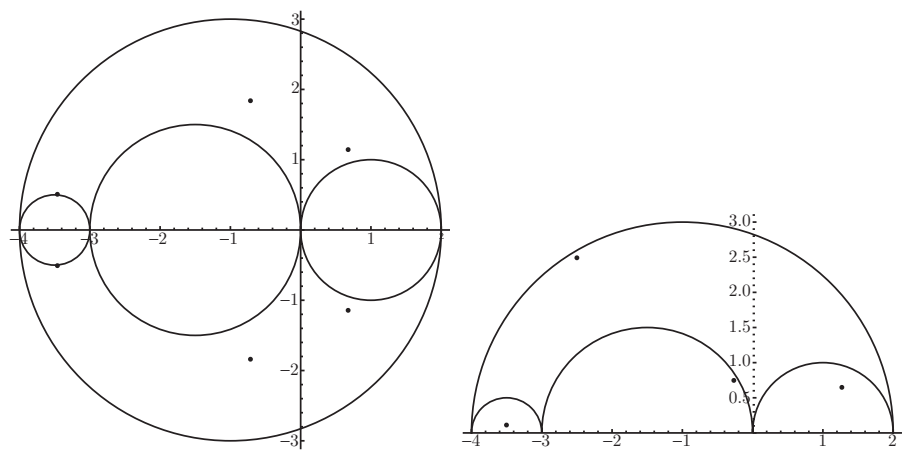

Figure 1. Critical points of $R=\frac{q}{p}$ and roots of $s=p-4 i q$ for $p=(z+4)(z+3) z(z-2)$ and $q=(z+7 / 2)(z+1)(z-1)$.

Theorem 1.3. In the above notation the open disk $D_{j}(\alpha)$ is a maximal real univalent disk of $R$.

The theory of univalent functions is a very classical domain closely related to the theory of quasiconformal mappings and Teichmüller spaces; see e.g. [6]. One of the central results, often called the criterion of univalency of functions in a disk, was proved by Z. Nehari in 1949. It claims that if the quotient of the absolute value of the Schwarzian derivative of $F$ by the square of the appropriate hyperbolic metrics in a disk is at most 2 , then $F$ is univalent. This result is also best possible within the whole class of holomorphic functions as shown by E. Hille, but it does not have to be sharp for individual functions. It is very tempting to understand the relation between Nehari's criterion and Theorem 1.3

\section{Proofs}

We first prove Theorem 1.1.

Proof. Denote by $p_{1}<q_{1}<p_{2}<q_{2}<\ldots<q_{k-1}<p_{k}$ the roots of $p$ and $q$ respectively. Since for any non-degenerate matrix $\left(\begin{array}{ll}a & b \\ c & d\end{array}\right)$ one has

$$
W(p, q)=(a d-b c) \cdot W(a p+b q, c p+d q),
$$

we can assume, without loss of generality, that $p=\prod_{j=1}^{k}\left(z-p_{j}\right)$ and that $q=$ $\prod_{j=1}^{k-1}\left(z-q_{j}\right)$.

Notice that the function $R=\frac{q}{p}$ can be represented as

$$
R(z)=\sum_{j=1}^{k} \frac{\alpha_{j}}{z-p_{j}}, \text { where } \alpha_{j}=\frac{q\left(p_{j}\right)}{p^{\prime}\left(p_{j}\right)}>0 .
$$

As we mentioned above the set of all roots of $W(p, q)$ coincides with that of the derivative

$$
R^{\prime}(z)=-\sum_{j=1}^{k} \frac{\alpha_{j}}{\left(z-p_{j}\right)^{2}} .
$$

The following lemma is a modification of [2, Theorem 2.5] and implies Theorem 1.1 . 
Lemma 2.1. All roots of $W(p, q)$ lie inside or on the above circle $C_{0}$.

Proof. Note that without loss of generality we can assume that $p_{1}+p_{k}=0$, since the statement is invariant under the addition of a real constant to the independent variable $z$. Assuming that $p_{1}+p_{k}=0$ set $\theta_{j}=\arg \left(z-p_{j}\right), j=1, \ldots, k$. Since $p$ and $q$ are real polynomials, we can restrict our attention to the case where $z$ lies in the upper half-plane and, therefore,

$$
0<\theta_{1}<\theta_{2}<\ldots<\theta_{k}<\pi .
$$

Now suppose that $|z|>\frac{p_{k}-p_{1}}{2}$, which implies that the origin lies outside the circle of radius $\frac{p_{k}-p_{1}}{2}$ centered at $z$. Therefore, $\theta_{k}-\theta_{1}<\pi / 2$, by the cosine rule. Since $\arg \left(z-p_{j}\right)^{-2}=-2 \theta_{j}$, then by (2.2) we get

$$
-2 \theta_{k} \leqslant \arg \left(z-p_{j}\right)^{-2} \leqslant-2 \theta_{1}, \quad j=1, \ldots, k,
$$

and, therefore, since all $\alpha_{j}>0$,

$$
2 \theta_{1} \leqslant \arg \frac{-\alpha_{j}}{\left(z-p_{j}\right)^{2}} \leqslant 2 \theta_{k}, \quad j=1, \ldots, k .
$$

Thus, since $\left|2 \theta_{k}-2 \theta_{1}\right|<\pi$, one has that all the numbers $\frac{-\alpha_{j}}{\left(z-p_{j}\right)^{2}}, j=1$, $\ldots, k$ lie on one side with respect to the straight line through the origin with the slope $\tan \left(2 \theta_{1}\right)$. Since $R^{\prime}(z)$ is the sum of these numbers, then $R^{\prime}(z) \neq 0$ at least for $|z|>\frac{p_{k}-p_{1}}{2}$.

Finally, notice that the assertion of Lemma 2.1 is invariant under Möbius transformations of $\mathbb{C} P^{1}$, since we can transform any pair of consecutive roots $\left(p_{j}, p_{j+1}\right)$ into the pair $\left(\tilde{p}_{1}, \tilde{p}_{k}\right)$ of the smallest and largest roots of another polynomial with the same properties by applying a suitable Möbius transformation. By (2.1) the roots of $W(p, q)$ do not change under any non-degenerate Möbius transformation and the claim follows.

Denoting by $p_{j}(\alpha)$ the roots of the polynomial $p+\alpha q$, we get $\lim _{\alpha \rightarrow+\infty} p_{j}(\alpha)=q_{j}$ for $j=1, \ldots, k-1$, and $\lim _{\alpha \rightarrow+\infty} p_{k}(\alpha)=+\infty$. Analogously, $\lim _{\alpha \rightarrow-\infty} p_{j}(\alpha)=$ $q_{j-1}$ for $j=2, \ldots, k$, and $\lim _{\alpha \rightarrow-\infty} p_{1}(\alpha)=-\infty$. Lemma 2.1 together with (2.1) implies that all zeros of the Wronskian $W(p, q)$ lie in the intersection of all circles centered at $\frac{p_{k}(\alpha)+p_{1}(\alpha)}{2}$ whose radii are equal to $\frac{p_{k}(\alpha)-p_{1}(\alpha)}{2}$.

Corollary 2.2. All roots of the Wronskian $W(p, q)$ lie in the intersection of the closed disk centered at $\frac{p_{k}+p_{1}}{2}$ of radius $\frac{p_{k}-p_{1}}{2}$ and the strip: $q_{1} \leqslant \operatorname{Im} z \leqslant q_{k-1}$.

To prove Proposition 1 let us first quote the main result of [10].

Theorem 2.3. Let $c_{1}, \ldots, c_{d}$ be a sequence of (not necessarily distinct) points in the open unit disk $D$ in the complex plane. Then there exists a unique proper holomorphic map $f: D \rightarrow D$ of degree $d+1$ normalized as $f(0)=0$ and $f(1)=1$ whose critical points are exactly $c_{1}, \ldots, c_{d}$. (If a certain point is repeated several times among $c_{1}, \ldots, c_{d}$, this means that the corresponding critical value has the corresponding multiplicity.) 


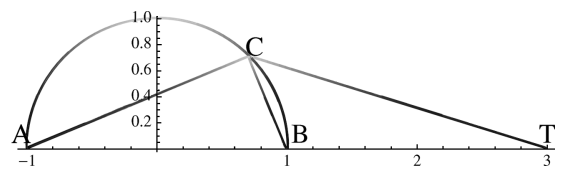

Figure 2. Illustration to the proof of Lemma 2.4.

To finish the proof of Proposition 1, note that a real rational function $R=\frac{q}{p}$ maps the open upper half-plane on the open upper half-plane if and only if $p$ and $q$ have all real, simple and interlacing roots and opposite signs of their leading coefficients. Since the upper half-plane can be mapped to the unit disk by a Möbius transformation we can arbitrarily prescribe $k-1$ critical points in the upper halfplane and find a degree $k$ map of the upper half-plane to itself with these (and no other) prescribed critical points. This map will be uniquely defined by Theorem 2.3 if we additionally use the restrictions as in Proposition 1

The next two propositions give interesting information on the behavior of $R$ on and inside the circle $C_{j}$ and (in our opinion) are of independent interest.

Proposition 2. In the above notation and for any $j=0, \ldots, k-1$ the function $|R|^{2}(z)$ restricted to the half-circle $C_{j}^{+}$is convex as a function of the real part of $z$ where $C_{j}^{+}$is the part of $C_{j}$ lying in the upper half-plane. In other words, $\Phi(x)=$ $|R|^{2}\left(x+i \sqrt{r_{j}^{2}-\left(x-\rho_{j}\right)^{2}}\right)$, where $x \in\left[p_{j}, p_{j+1}\right], r_{j}=\frac{p_{j+1}-p_{j}}{2}, \rho_{j}=\frac{p_{j+1}+p_{j}}{2}$ is convex as a function of $x$.

We will first prove convexity of $|R|^{2}$ with respect to a more convenient coordinate on $C_{j}^{+}$. Denote temporarily by $A$ and $B$ the roots $p_{j}$ and $p_{j+1}$ respectively and denote by $C$ an arbitrary point of the half-circle $C_{j}^{+}$. Let $w:=|B C|^{2}$ be the square of the length of the segment $B C$. Obviously, $w$ varies from 0 to $\left(p_{j+1}-p_{j}\right)^{2}$ when $C$ runs along $C_{j}^{+}$from $p_{j+1}$ to $p_{j}$. Let us express the function $|R|^{2}$ in terms of $w$.

Lemma 2.4. One has

$$
|R|^{2}(w)=\frac{\prod_{l=1}^{k-1}\left(s_{l} w+t_{l}\right)}{\prod_{l=1}^{k}\left(u_{l} w+v_{l}\right)}
$$

where $t_{l}=\left(q_{l}-p_{j+1}\right)^{2}, v_{l}=\left(p_{l}-p_{j+1}\right)^{2}, s_{l}=\frac{2 q_{l}-p_{j}-p_{j+1}}{p_{j+1}-p_{j}}, v_{l}=\frac{2 p_{l}-p_{j}-p_{j+1}}{p_{j+1}-p_{j}}$.

Proof. Take an arbitrary point $T$ on the real axis (assume for the moment that $T>$ $p_{j+1}$ ) and consider the triangle $A C T$; see Figure 2, By the well-known Stewart's theorem from elementary geometry applied to the triangle $A C T$ with a Cevian $B C$, one gets

$$
|B C|^{2}=\frac{|A B|}{|A T|}|C T|^{2}+\frac{|B T|}{|A T|}|A C|^{2}-|A B||B T| .
$$

Since $|A C|^{2}=|A B|^{2}-|B C|^{2}$ the latter formula is equivalent to

$$
|C T|^{2}=\frac{2 T-A-B}{B-A}|B C|^{2}+(B-T)^{2}=\frac{(2 T-A-B) w}{B-A}+(B-T)^{2} .
$$

One can easily check that (2.4) is valid even if $T \leqslant p_{j+1}$, which implies (2.3). 
Remark 2.5. Notice that although we are primarily interested in $|R|^{2}(w)$ when $w$ belongs to the interval $\left(0,\left(p_{j+1}-p_{j}\right)^{2}\right)$ the formula (2.3) defines $|R|^{2}(w)$ globally as a rational function of $w$, which we will be using below.

Proof of Proposition 2. Invariance under an affine change of $z$ allows us to assume, without loss of generality, that $p_{j}=-1$ and $p_{j+1}=1$, which leads to the formula

$$
|R|^{2}(w)=\frac{\prod_{l=1}^{k-1}\left(q_{l} w+\left(1-q_{l}\right)^{2}\right)}{\prod_{l=1}^{k}\left(p_{l} w+\left(1-p_{l}\right)^{2}\right)} .
$$

If we denote by $\xi$ any of the points $q_{l}$ (resp. $p_{l}$ ), then the corresponding linear factor in the numerator (resp. denominator) has the form $\xi w+(1-\xi)^{2}$. Its only root is given by the formula $\zeta=\Psi(\xi)=-\frac{(1-\xi)^{2}}{\xi}$. The rational map $\Psi(\xi)$ is a double covering of $\mathbb{R} P^{1} \backslash(0,4)$ by $\mathbb{R} P^{1}$, namely, the interval $(+0,1]$ is mapped to $(-\infty, 0]$, the interval $[1,+\infty)$ is mapped to $[0,-\infty)$; the interval $(-\infty,-1]$ is mapped to $(+\infty, 4]$; and, finally, the interval $[-1,-0)$ is mapped to $[4,+\infty)$. See Figure 3. This observation implies that if we consider the images of our roots $p_{1}<q_{1}<\ldots<p_{j}=-1<q_{j}<p_{j+1}=1<\ldots<q_{k-1}<p_{k}$ under the map $\Psi$, then $p_{j+1}$ will be mapped to 0 , and $q_{j+1}<\ldots<p_{k}$ will be mapped to the negative half-axis in the order-reversing manner. Analogously, $p_{j}$ is mapped to 4 , and all roots $p_{1}<q_{1}<\ldots<q_{j-1}$ will be mapped to $(4, \infty)$ in the order-reversing manner. The only root $q_{j} \in(-1,1)$ can be mapped completely arbitrarily to $\mathbb{R} P^{1} \backslash[0,4]$. In particular, if $q_{j}=0$, then $\Psi\left(q_{j}\right)=\infty$. Thus, considering $|R|^{2}(w)$ as a function on $\mathbb{R} P^{1}$, we obtain that exactly $k-2$ of a total of $k$ intervals between consecutive roots of the denominator of (2.5) contain exactly one root of the numerator. The remaining two intervals will be called exceptional. One of these exceptional intervals is $(0,4)$, where there are no roots of the numerator. The other exceptional interval is the one containing $\Psi\left(q_{j}\right)$. Besides $\Psi\left(q_{j}\right)$ it contains one more root of the numerator (counting multiplicities) as shown in Figure 3. (Recall that the numerator and denominator of (2.5) are to be considered as homogeneous polynomials of degree $k$ in homogeneous coordinates on $\mathbb{R} P^{1}$; i.e., the numerator always has a root at $\infty$.) Notice that it might happen that $\Psi\left(q_{j}\right)=\Psi\left(p_{i}\right),(i \neq j, j+1)$. In this case $\operatorname{deg}|R|^{2}(w)$ decreases by 1 , but the required statement still holds.

The function $|R|^{2}(w)$ restricted to each closed non-exceptional interval on $\mathbb{R} P^{1}$ maps it bijectively to the whole $\mathbb{R} P^{1}$. Consider now the behavior of $|R|^{2}(w)$ on $(0,4)$. It is strictly positive there and tends to $+\infty$ when $w \rightarrow 0+$ or $w \rightarrow 4-$. One can immediately see that there can be at most two solutions to the equation $|R|^{2}(w)=a w+b$ on $(0,4)$, where $a, b$ are arbitrary real numbers. Indeed, consider the equation $|R|^{2}(w)=a w+b$ globally on $\mathbb{R} P^{1}$. Notice that unless $\Psi\left(q_{j}\right)=$ $\Psi\left(p_{i}\right), \quad(i \neq j, j+1)$ the degree of $|R|^{2}(w)$ as a rational function equals $k$ and, therefore, the latter equation can have at most $k$ real solutions on the whole $\mathbb{R} P^{1}$, counting multiplicities. We already know that under the same assumption this equation has exactly one real solution on each of the $k-2$ non-exceptional intervals. Thus, there can be at most 2 solutions on $(0,4)$; see Figure 3 . Finally, notice that a smooth positive function defined on $(0,4)$, tending to $+\infty$ on both ends and whose graph intersects any straight line at most twice (counting multiplicities) is convex. Thus, we have proved that $|R|^{2}(w)$ is a convex function of $w \in(0,4)$. (The exceptional case $\Psi\left(q_{j}\right)=\Psi\left(p_{i}\right),(i \neq j, j+1)$ is considered in exactly the same manner.) To prove that $|R|^{2}(x)$ is convex, where $x$ is the real part of $z$ on the upper half-circle of the unit circle, notice that $w=2(1-x)$. The latter relation is 


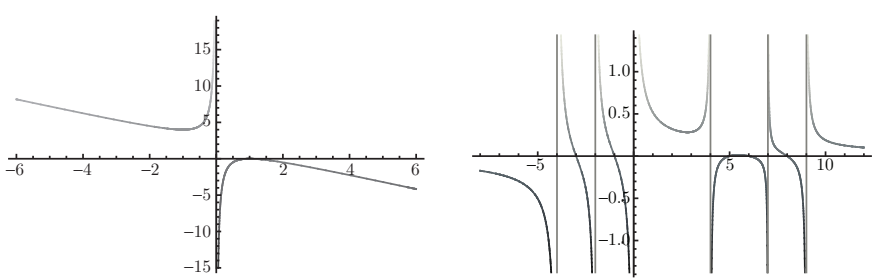

FiguRE 3. $\Psi(\xi)=-\frac{(1-\xi)^{2}}{\xi}$ and an example of $|R|^{2}(w), w \in \mathbb{R}$.

easily obtained from the Pythagorean theorem. Since convexity is preserved under affine changes of coordinates, the result follows.

Now consider the family of level curves $\Gamma_{r}:\left\{|R|^{2}(z)=r^{2} \geqslant 0\right\}$ and intersect all curves in this family with a given disk $D_{j}$; see Figure 4. Call a positive number $r$ non-critical for $R(z)$ if it is not the modulus of some of its critical values. Notice that if $r>0$ is non-critical, then the curve $\Gamma_{r}$ consists of a number of smooth ovals and it is invariant under complex conjugation. Set $m=\min _{z \in C_{j}^{+}}|R|(z)$. Notice that by Proposition 2 the value $m$ is attained at the unique point $P$ on $C_{j}^{+}$.

Proposition 3. For any $0<r \leqslant m$ the intersection of the level curve $\Gamma_{r}$ : $|R|^{2}(z)=r^{2}>0$ with $\bar{D}_{j}$ is a single oval containing $q_{j}$, where $\bar{D}_{j}$ is the closure of $D_{j}$. For any $r>m$ this intersection is a pair of arcs each crossing the interval $\left(p_{j}, p_{j+1}\right) \subset \mathbb{R}$.

Proof. Let us first show that for any non-critical $r$ the curve $\Gamma_{r} \subset \mathbb{C} P^{1}$ consists of exactly $k$ smooth ovals each of which intersects $\mathbb{R}$ in two points and is, therefore, invariant under complex conjugation. Notice that the function $R(z)$ induces a $k$ fold covering of the circle of radius $r$ by $\Gamma_{r}$. For a non-critical $r$ the curve $\Gamma_{r}$ consists of at most $k$ smooth ovals and is invariant under conjugation. Each such oval is either self-conjugate and thus intersects $\mathbb{R}$ or does not intersect $\mathbb{R}$, and then it has a complex conjugate pair. Observe that the function $|R|^{2}(z)$ restricted to $\mathbb{R}$ coincides with $R^{2}(z)$ and is a real non-negative rational function of degree $2 k$. Since $|R|^{2}(z)$ vanishes at each $q_{j} \in\left(p_{j}, p_{j+1}\right)$ and tends to $+\infty$ when $z \rightarrow p_{j}$, one can easily observe that it is convex on each $\left(p_{j}, p_{j+1}\right)$, implying that for any $r>0$ the curve $\Gamma_{r}$ intersects $\mathbb{R}$ exactly $2 k$ times and, thus, consists of $k$ self-conjugate ovals for any non-critical $r$.

Notice that singularities of $\Gamma_{r}, r>0$ can only occur at the critical points of $R(z)$. Thus, since $D_{j}$ contains no critical points of $R(z)$, any curve $\Gamma_{r}, r>0$ restricted to $D_{j}$ is non-singular. The function $|R|^{2}(z)$ is non-negative in $\mathbb{C}$ and vanishes only at the roots $q_{1}<\ldots<q_{k-1}$. Thus, it vanishes only once inside $D_{j}$, namely, at $q_{j}$. Therefore, for sufficiently small $r>0$ the curve $\Gamma_{r}$ intersected with $D_{j}$ is a small oval around $q_{j}$. Again due to the absence of critical points inside $D_{j}$ this family of ovals persists until $r$ reaches the value $m$. The oval $O_{m}$ corresponding to $r=m$ will be tangent to the circle $C_{j}$; see Figure 4. Indeed, since $m=\min |R|^{2}(z)$ on $C_{j}^{+}$the oval $O_{m}$ has to have a common point $P$ with $C_{j}^{+}$. The latter point $P$ is unique due to convexity of $|R|^{2}(z)$ on $C_{j}^{+}$with respect to the real part of $z$, and since $O_{m}$ is smooth, $P$ is the tangency point of $O_{m}$ and $C_{j}^{+}$. The remaining part of $D_{j}$ consists of two crescents each of which will be covered by a family of arcs 


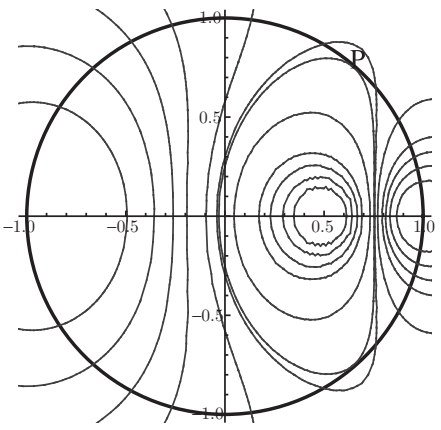

FiguRE 4. Level curves of $|R|^{2}(z)$ within the disk $|z| \leqslant 1$ where $R(z)=\frac{(z-1 / 2)(z-2)(z+2)}{(z-1)(z+1)(z-3)(z+3)}$.

formed by intersecting $\Gamma_{r}, r>m$ with $D_{j}$. Indeed, denote the intersection points of $O_{m}$ with $\mathbb{R}$ as $a<b$. Note that $|R(z)|$ restricted to $C_{j}^{+}$is strictly monotone decreasing from $+\infty$ to $m$ and then strictly monotone increasing back to $+\infty$ when $z$ moves along $C_{j}^{+}$from $p_{j}$ to $p_{j+1}$. Thus we get that for each $r>m$ there exist a unique point in $\left(p_{j}, a\right)$ and a unique point on the part on $C_{j}^{+}$from $p_{j}$ to $P$ such that $|R(z)|=r$. A similar pair of points exists on $\left(b, p_{j+1}\right)$ and the part of $C_{j}^{+}$from $P$ to $p_{j+1}$ respectively. The curve $\Gamma_{r}$ must necessarily connect the first pair of points and the second pair of points. Indeed, if we follow $\Gamma_{r}$ from the point of $\mathbb{R}$, then it cannot stay within $D_{j}$ since it cannot return back to the real axis. Moreover, it can only leave $D_{j}$ at the unique point on $C_{j}^{+}$due to the convexity of $|R|^{2}(z)$ on $C_{j}^{+}$ w.r.t. the real part of $z$.

Let us finally settle Theorem 1.3 .

Proof. A maximal open contractible univalent domain $D \subset \mathbb{C} P^{1}$ of a holomorphic function $F$ has the property that $F$ is injective on $D$, but some points on the boundary of $D$ have the same image. In our case the image under $R$ of the real interval $\left[p_{j}, p_{j+1}\right]$ is the whole $\mathbb{R} P^{1}$ with $\infty$ covered twice by $p_{j}$ and $p_{j+1}$. Analogously, the image under $R$ of the half-circle $C_{j}^{+}$is a closed curve starting and ending at $\infty$. If we show that $R\left(C_{j}^{+}\right)$is a simple loop in $\mathbb{C} P^{1}$ non-intersecting $\mathbb{R} P^{1}$ at points other than $\infty$, then Theorem 1.3 will easily follow. Indeed, in this case the open half-disk $D_{j}^{+}$bounded by $\left[p_{j}, p_{j+1}\right]$ and $C_{j}^{+}$will be mapped bijectively to the part of the lower half-plane $\Im z<0$ lying above the loop $R\left(C_{j}^{+}\right)$. Analogously, the open half-disk $D_{j}^{-}$ bounded by $\left[p_{j}, p_{j+1}\right]$ and $C_{j}^{-}$will be mapped bijectively to the part of the upper half-plane $\Im z>0$ lying below the loop $R\left(C_{j}^{-}\right)$. (Indeed, our domain is a disk which is mapped locally diffeomorphically on its image, and its boundary is mapped onto the union of 2 closed smooth loops having a single common point.) The fact that $R\left(C_{j}^{+}\right)$is a simple loop is almost true except for the special case when $\operatorname{deg} R=2$. Notice that any function $R$ as in (1.1) restricted to $\mathbb{R} P^{1}$ induces a $k$-fold covering of $\mathbb{R} P^{1}$ by $\mathbb{R} P^{1}$. Since $\operatorname{deg} R=k$ there are no points in $\mathbb{C} P^{1} \backslash \mathbb{R} P^{1}$ whose image under $R$ is real. Therefore, no points from $C_{j}^{+}$except for its endpoints are mapped to $\mathbb{R} P^{1}$. To prove that $R\left(C_{j}^{+}\right)$is a simple loop for $\operatorname{deg} R>2$, notice that our statement 
is invariant under the action of the Möbius group on $R$ and we can assume, without loss of generality, that $p_{1}=-1<q_{1}=0<p_{2}=1<q_{2}<p_{3}<\ldots<q_{k-1}<p_{k}$.

Lemma 2.6. Under the above assumptions and if $\operatorname{deg} R(z)>2$, then the function $\Re R(z)$ is monotone decreasing when restricted to $C_{1}^{+}$parameterized from $p_{1}$ to $p_{2}$. In the special case $\operatorname{deg} R(z)=2$, the function $\Re R(z)$ is constant. (Here $\Re F$ means the real part of $F$.)

Proof. Let us express $\Re R(x)$ explicitly. Recall that

$$
R(z)=\sum_{j=1}^{k} \frac{\alpha_{j}}{z-p_{j}}=\frac{\alpha_{1}}{z+1}+\frac{\alpha_{2}}{z-1}+\sum_{j=3}^{k} \frac{\alpha_{j}}{z-p_{j}},
$$

where $\alpha_{j}>0$ and $p_{j}>1$ for $j>2$. Then substituting $z=x+i y$ one gets

$$
\Re R(x+i y)=\frac{\alpha_{1}(x+1)}{(x+1)^{2}+y^{2}}+\frac{\alpha_{2}(x-1)}{(x-1)^{2}+y^{2}}+\sum_{j=3}^{k} \frac{\alpha_{j}\left(x-p_{j}\right)}{\left(x-p_{j}\right)^{2}+y^{2}} .
$$

To show that $\Re R$ is monotone decreasing on the upper half of the unit circle let us parameterize it by $x \in[-1,1] ; y=\sqrt{1-x^{2}}$. Then

$$
\begin{aligned}
& \Re R(x)=\frac{\alpha_{1}(x+1)}{(x+1)^{2}+\left(1-x^{2}\right)}+\frac{\alpha_{2}(x-1)}{(x-1)^{2}+\left(1-x^{2}\right)}+\sum_{j=3}^{k} \frac{\alpha_{j}\left(x-p_{j}\right)}{\left(x-p_{j}\right)^{2}+\left(1-x^{2}\right)} \\
& =\frac{\alpha_{1}}{2}-\frac{\alpha_{2}}{2}+\sum_{j=3}^{k} \frac{\alpha_{j}\left(x-p_{j}\right)}{\left(x-p_{j}\right)^{2}+\left(1-x^{2}\right)} .
\end{aligned}
$$

Notice that each summand $\Psi_{j}(x)=\frac{\alpha_{j}\left(x-p_{j}\right)}{\left(x-p_{j}\right)^{2}+\left(1-x^{2}\right)}$ is a monotone decreasing function for $\alpha_{j}>0, p_{j}>1, x \in[-1,1]$. Indeed, a simple calculation shows that

$$
\Psi_{j}^{\prime}(x)=\alpha_{j} \frac{1-p_{j}}{\left(1+p_{j}^{2}-2 p_{j} x\right)^{2}}<0
$$

Thus, we conclude that since $\Re R$ is a monotone decreasing function the curve $R\left(C_{j}^{+}\right)$is a simple loop starting and ending at $\infty$ and not intersecting $\mathbb{R} P^{1}$. Theorem 1.3 is settled for $\operatorname{deg} R>2$. In the case that $\operatorname{deg} R=2$ one can easily see that $R\left(C_{j}^{+}\right)$is a double covering of a vertical ray in $\mathbb{C}$ lying below the real axis.

Corollary 2.7. The number $m=\min _{z \in C_{j}^{+}}|R|(z)$ is smaller than the least modulus among the critical values of $R(z)$.

Finally, let us come back to the Hermite-Biehler theorem, which states that, for given monic real polynomials $p$ and $q$ of degrees $k$ and $k-1$, respectively, that the polynomial

$$
s(z)=p(z)+i \alpha q(z)
$$

has all zeroes in the upper half-plane if and only if $\alpha<0$ and the polynomials have real, simple and interlacing zeroes. More generally, by the Hermite-Biehler theorem, all roots of the equation

$$
\frac{q(z)}{p(z)}=r e^{i \varphi}, \quad r>0, \varphi \in(-\pi, \pi]
$$


lie in the upper (resp. lower) half-plane if and only if the polynomials have real, simple and interlacing zeroes and $\varphi \in(-\pi, 0)$ (resp. $\varphi \in(0, \pi)$ ). All roots of the equation (2.6) are real and interlace both zeroes of the polynomials $p$ and $q$ if and only if $\varphi$ equals 0 or $\pi$.

Theorem 1.3 allows us to locate the roots of the equation (2.6) more precisely and, therefore, to improve the Hermite-Biehler theorem. In fact, Theorem 1.3 implies the following corollary.

Corollary 2.8. Each open disk $D_{j}, j=1, \ldots, k$, as well as the open disk $\mathbb{C} \backslash \bar{D}_{0}$ on $\mathbb{C} P^{1}$ contains at most one root of the equation (2.6).

Considering the equation (2.6) as an equation on $\mathbb{C}$, we obtain from Corollary 2.8 the following statement.

Corollary 2.9. All solutions of the equation (2.6) except at most one lie in the closed disk $\bar{D}_{0}$.

\section{SOME OPEN PROBLEMS}

1. Describe the boundary of $\bigcap_{\alpha \in \mathbb{R}} \Omega_{\alpha}$ and find its algebraic equation.

2. Is Nehari's criterion (see introduction) satisfied for disks $D_{j}(\alpha)$ ?

3. Are there other classes of real rational functions for which the univalency of restriction guarantees the univalency in the disk? In particular, what happens to real rational functions with all real roots and poles?

4. Find an analog of Theorem 1.3 for pairs of real-rooted polynomials $(p, q)$ satisfying the assumptions of Dedieu's theorem; see [1], in which case the straight segment connecting $p$ and $q$ consists of real-rooted polynomials.

5. Conjecture. For any $R$ as in (1.1) the loop $R\left(C_{j}^{+}\right)$is a convex curve on $\mathbb{C}$.

\section{ACKNOWLEDGEMENTS}

The first and third authors want to acknowledge the hospitality of the mathematics department of Stockholm University in October 2009 and January 2010. The authors want to thank A. Eremenko for relevant explanations of the main result of [10] and P. Bränden for important discussions on the topic.

\section{REFERENCES}

1. J. P. Dedieu, Obreschkoff's theorem revisited: What convex sets are contained in the set of hyperbolic polynomials?, J. Pure and Appl. Algebra (1992), 269-278. MR.1179101|(93g:12001)

2. K. Dilcher, K. B. Stolarsky, Zeros of the Wronskian of a polynomial, J. Math. Anal. Appl. 162 (1991), 430-451. MR1137630 (92m:30008)

3. A. Eremenko, A. Gabrielov, Degrees of real Wronski maps. Discrete Comput. Geom. 28 (2002), no. 3, 331-347. MR1923956 (2003g:14074)

4. A. Eremenko, A. Gabrielov, The Wronski map and Grassmannians of real codimension 2 subspaces. Comput. Methods Funct. Theory 1 (2001), no. 1, 1-25. MR.1931599(2003h:26022)

5. S. Fisk, Polynomials, roots, and interlacing, arXiv:math/0612833.

6. O. Lehto, Univalent Functions and Teichmüller Spaces, Grad. Texts Math., Vol. 109, SpringerVerlag, 1986. MR867407 (88f:30073)

7. M. Marden, Geometry of Polynomials, Math. Surveys, no. 3, Amer. Math. Soc., Providence, RI, 1966. MR0225972 (37:1562)

8. Z. Nehari, The Schwarzian derivative and schlicht functions. Bull. Amer. Math. Soc. 55 (1949), 545-551. MR0029999 (10:696e) 
9. Q. I. Rahman, G. Schmeisser, Analytic theory of polynomials, London Math. Soc. Monogr. (N. S.), Vol. 26, Oxford Univ. Press, New York, NY, 2002. MR.1954841 (2004b:30015)

10. S. Zakeri, On critical points of proper holomorphic maps on the unit disk. Bull. London Math. Soc. 30 (1998), no. 1, 62-66. MR1479037 (99d:30009)

Laboratoire de Mathématiques, Université de Nice, Parc Valrose, 06108 Nice Cedex 2, FRANCE

E-mail address: kostov@unice.fr

Department of Mathematics, Stockholm University, SE-106 91, Stockholm, Sweden

E-mail address: shapiro@math.su.se

Institut für Mathematik, MA 4-5 Technische Universität Berlin, D-10623 Berlin, GERMANY

E-mail address: tyaglov@math.tu-berlin.de 